\title{
Eu/RG absorption and excitation spectroscopy in the solid rare gases: State dependence of crystal field splitting and Jahn-Teller coupling
}

\author{
Owen Byrne and John G. McCaffreya) \\ Department of Chemistry, National University of Ireland, Maynooth, County Kildare, Ireland
}

(Received 8 November 2010; accepted 22 February 2011; published online 22 March 2011)

\begin{abstract}
Absorption spectroscopy recorded for annealed samples of matrix-isolated atomic europium reveals a pair of thermally stable sites in Ar and $\mathrm{Kr}$ while a single site exists in Xe. Plots of the matrix shifts of the visible $\mathrm{s} \rightarrow \mathrm{p}$ bands versus host polarizability, allowed the association of the single site in Xe and the blue sites in $\mathrm{Ar}$ and $\mathrm{Kr}$. On the basis of the similar ground state bond lengths expected for the Eu-rare gas (RG) diatomics and the known Na-RG molecules, the blue sites are attributed to Eu occupancy in the smaller tetra-vacancy while the red sites are proposed to arise from hexa-vacancy sites. Both sites are of cubic symmetry, consistent with the pronounced Jahn-Teller structure present on the $y^{8} \mathrm{P} \leftarrow a^{8} \mathrm{~S}_{7 / 2}$ transition for these bands in the three hosts studied. Site-selective excitation spectroscopy has been used to reanalyze complex absorption spectra previously published by Jakob et al. [Phys. Lett. A 57, 67 (1976)] for the near-UV $\mathrm{f} \rightarrow \mathrm{d}$ transitions. On the basis that a pair of thermally stable sites exist in solid argon, the occurrence of crystal field splitting has been identified to occur for the $J \geq 5 / 2$ level of the ${ }^{8} \mathrm{P}$ state when isolated in these two sites with cubic symmetry. From a detailed lineshape analysis, the magnitude of the crystal field splittings on the $J=5 / 2$ level in $\mathrm{Ar}$ is found to be 105 and $123 \mathrm{~cm}^{-1}$ for the red and blue sites, respectively. (C) 2011 American Institute of Physics. [doi:10.1063/1.3564947]
\end{abstract}

\section{INTRODUCTION}

Previous work by the Maynooth group has focused on the luminescence of the metal atoms cadmium, ${ }^{1}$ zinc, ${ }^{2}$ mercury, ${ }^{3}$ and magnesium, ${ }^{4}$ and, more recently, sodium, ${ }^{5}$ and manganese $\mathrm{e}^{6-8}$ isolated in the solid rare gases (RG's). These systems possess spherically symmetric $n \mathrm{~s}^{1}$ or $n \mathrm{~s}^{2} \mathrm{~S}$ ground states and involve $n \mathrm{p}^{1}$ or $n \mathrm{~s}^{1} n \mathrm{p}^{1}$ excited states which can be reached in fully allowed absorptions in the UV-visible (Vis) spectral region. Mn, the most recent metal studied, ${ }^{7}$ is located in the centre of the transition metal series and with a halffull d-orbital, yields a ${ }^{6} \mathrm{~S}$ ground state from the $[\mathrm{Ar}] 3 \mathrm{~d}^{5} 4 \mathrm{~s}^{2}$ configuration. Spherically symmetric metal atoms have been selected for study because of the isotropic interactions the ground states of these atoms have with their surroundings in the $f c c$ solid rare gases. Because of the relatively simple nature of these high symmetry interactions theoretical studies, involving a comparison of the recorded optical spectroscopy with the results of molecular dynamics simulations, are achievable. Ultimately, the existence of a pair of thermally stable sites in each of the following systems $\mathrm{Na}(\mathrm{s})^{1}$, $\mathrm{Mn}(\mathrm{d})^{5}$, and as will now be presented for $\operatorname{Eu}(\mathrm{f}),{ }^{7}$ is amenable to straightforward investigation with theoretical means once reliable metal (M)-RG interaction potentials are available. This has already been done in the case of matrix-isolated atomic sodium ${ }^{5}$ where multiple sites are known to be occupied. In contrast, non-S ground state atoms present complications where several closely lying levels exist either from numerous electronic configurations ${ }^{9}$ or static Jahn-Teller (JT) splitting, best exemplified by $\mathrm{P}$ states of the halogens. ${ }^{10}$

\footnotetext{
a)Electronic mail: john.mccaffrey@nuim.ie.
}

Having studied group I, II, and transition metal atom systems, the next logical extension is to investigate matrixisolated rare earth (lanthanide) metals. With a half-filled forbital, the lanthanide equivalent of manganese is europium. Its lowest energy [Xe $] 4 \mathrm{f}^{7} 6 \mathrm{~s}^{2}$ electronic configuration is spherically symmetric so an ${ }^{8} \mathrm{~S}$ ground state results and similar to the previous systems studied by us, it has an excited $6 s^{1} 6 p^{1}\left(y^{8} \mathrm{P}\right)$ electronic state accessible in absorption in the visible spectral region. The aforementioned similarities between $\mathrm{Mn}$ and Eu led to europium being chosen as the system of choice. However, relatively little spectroscopy has been presented in the literature for matrix-isolated lanthanide atoms. To the best of our knowledge, only the luminescence of the $\mathrm{Tm} / \mathrm{Ne}$ (Ref. 11) system has been thoroughly investigated. Some work also exists from the 1970s on the core $\mathrm{f} \rightarrow$ d type UV absorptions of matrix-isolated Eu (Refs. 12 and 13) and limited studies have been done on the Yb/RG (Ref. 14) and Ho/RG (Ref. 15) systems. Thus, it appears that the spectroscopy of the matrix-isolated lanthanide metal atoms has not received the attention it deserves, and it is an important topic worthy of exploration and analysis.

The near-UV absorptions, arising from the $\mathrm{f} \rightarrow \mathrm{d}$ type ${ }^{8} \mathrm{P}$ $\leftrightarrow a^{8} \mathrm{~S}$ electronic transitions of $\mathrm{Eu} / \mathrm{RG}$, were identified and investigated previously by Jakob et al. ${ }^{12}$ in samples formed at $4 \mathrm{~K}$ and annealed up to $30 \%$ of the hosts' melting point. Spectra were recorded at high resolution $\left(10 \mathrm{~cm}^{-1}\right)$ revealing structured but very complex absorption features. Jakob et al. suggested that the complex structure of the absorption spectrum was a result of crystal field (CF) splitting of the ${ }^{8} \mathrm{P}_{\mathrm{J}}$ states. In this earlier work, Eu was assumed to exist in a single site of noncubic symmetry in the RG lattices. With the ability to perform site-specific excitation spectra and our 


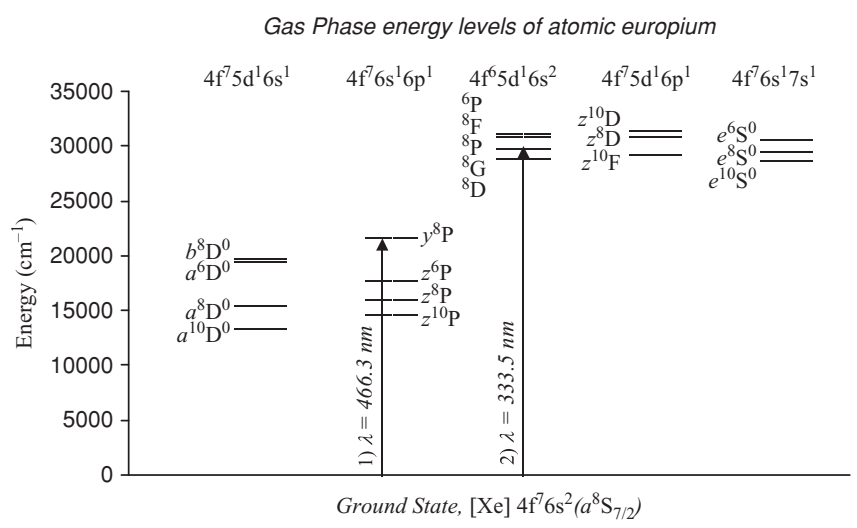

FIG. 1. Schematic representation of the energy levels (Ref. 16) of gas phase atomic europium. The $y^{8} \mathrm{P}_{5 / 2} \leftarrow a^{8} \mathrm{~S}_{7 / 2}$ and ${ }^{8} \mathrm{P}_{5 / 2} \leftarrow a^{8} \mathrm{~S}_{7 / 2}$ transitions which have the largest absorption oscillator strengths occur at $21444.6 \mathrm{~cm}^{-1}$ $(466.3 \mathrm{~nm})$ and $29982.5 \mathrm{~cm}^{-1}(333.5 \mathrm{~nm})$, respectively, are indicated by the arrows.

current knowledge of the sites of isolation of atomic Eu in the RG's, it is now possible to review the previously studied UV absorption features. By conducting a detailed lineshape analysis of the site-resolved bands, a more realistic CF model of the complex splitting observed can be developed.

Figure 1 presents a complete gas phase ${ }^{16}$ energy level diagram for the UV-Vis absorption transitions of atomic Eu. With an $a^{8} \mathrm{~S}$ ground state, two absorptions are observed in the gas phase for atomic Eu with large oscillator strengths, viz., the $y^{8} \mathrm{P}$ state in the visible with its lowest spin-orbit level occurring at $466.3 \mathrm{~nm}$ and the near-UV ${ }^{8} \mathrm{P}$ state at $333.5 \mathrm{~nm}$. In the gas phase the $y^{8} \mathrm{P}$ transition from the $a^{8} \mathrm{~S}_{7 / 2}$ ground state is ten times stronger than the near-UV ${ }^{8} \mathrm{P} \leftarrow a^{8} \mathrm{~S}_{7 / 2}$ transition. To date, however, no work has appeared in the literature pertaining to the visible absorption of matrix-isolated $\mathrm{Eu}$. In addition a previously undocumented $6 s^{1} 6 \mathrm{p}^{1} \mathrm{z}^{6} \mathrm{P}$ state will be analyzed in the newly obtained excitation spectra. The present study aims to identify and probe these electronic states using a combination of observed matrix shifts and with estimates of the Eu-RG nearest neighbor distances, several site occupancies will be considered and discussed.

\section{EXPERIMENTAL}

The gas handling system and vacuum apparatus used in the preparation of the Eu/RG matrix samples have been described in previous works ${ }^{3}$ from our group. Europium vapor was generated by electron bombardment of Eu metal chips (Sigma Aldrich, 457965-5G, 99.9\% purity) contained in a molybdenum crucible. Samples were deposited at gas flow rates of $3 \mathrm{mmol} / \mathrm{h}$ for periods of $30 \mathrm{~min}$. Concentration studies were conducted by keeping these deposition parameters fixed while varying the metal flow as indicated on the flux monitor on the Omicron (model EFM3 UHV) evaporator. The atomic excitation and absorption spectra reported in this study were recorded in the most dilute $\mathrm{Eu} / \mathrm{RG}$ samples formed. Samples were annealed to 26, 36, and $60 \mathrm{~K}$ for $\mathrm{Ar}, \mathrm{Kr}$, and $\mathrm{Xe}$, respectively, to simplify the site occupancy. Sample temperature was measured with a Si diode, mounted on the copper sample holder, while it was monitored and set with a LakeShore
Cryogenics (model 321 autotuning) temperature controller. Separate samples were prepared at elevated temperatures to enhance the formation of Eu clusters.

The experimental setups used for recording absorption and excitation spectra have been described in detail elsewhere. ${ }^{3}$ Continuous lamp excitation scans were obtained either from a deuterium lamp (UV, 180-400 nm range) or a tungsten lamp (UV-Vis, 300-900 nm range). The excitation wavelength was selected using a $0.3 \mathrm{~m}$ focal length monochromater (Acton Research Corporation, model SpectraPro-300i) containing a 1200 grooves $/ \mathrm{mm}$ diffraction grating blazed at $300 \mathrm{~nm}$. Absorption spectra were recorded directly through the matrix sample using a Hamamatsu R928 PMT detector, while excitation spectra were monitored perpendicular to the light source. Excitation spectra were recorded with monochromator slits of $0.1 \mathrm{~mm}$ while slits of $10 \mu \mathrm{m}$ were used for absorption scans. These settings provide spectral resolution of 1.0 and $0.1 \mathrm{~nm}$, respectively, with the $1200 \mathrm{~g} / \mathrm{mm}$ grating used in the $0.3 \mathrm{~m}$ focal length monochromater.

The emission wavelength of interest was selected using a $0.5 \mathrm{~m}$ Spectra-Pro-500i monochromater fitted with three gratings and the emission intensity was monitored using a Hamamatsu R928-P PMT detector held at $-20^{\circ} \mathrm{C}$ in a Photocool S600 cooling housing. Laser excitation spectra were recorded by scanning the output of a Nd:YAG pumped Quantel TDL-90 dye laser in the region of the $z^{6} \mathrm{P} \leftarrow a^{8} \mathrm{~S}$ transition using Rhodamine $590(6 \mathrm{G})$ as dye material. In this case an intensified gated-iCCD detector (Andor, model DH720), mounted on the $0.5 \mathrm{~m}$ monochromater (ARC, SP500i) was used to monitor the emission intensity.

\section{RESULTS}

\section{A. Absorption spectroscopy}

Atomic europium was readily isolated in the RG (RG $=\mathrm{Ar}, \mathrm{Kr}$, and $\mathrm{Xe}$ ) matrices at $10 \mathrm{~K}$. The absorption spectra recorded for annealed $\mathrm{Eu} / \mathrm{RG}$ samples in the UV-Vis spectral region are presented in Fig. 2. Two main absorption regions are clearly identifiable. An intense, broad absorption is observed in the visible region and three weaker, narrow features occur in the near-UV region. The dashed vertical lines in Fig. 2 indicate the gas phase ${ }^{16}$ positions of the $y^{8} \mathrm{P}$ and ${ }^{8} \mathrm{P}$ state absorptions. Based on their spectral positions and splitting patterns, the near-UV features are confidently attributed to resolved spin-orbit levels of the ${ }^{8} \mathrm{P}_{\mathrm{J}}$ state. Figure 2 reveals complex structures centered at approximately $450 \mathrm{~nm}$ and considerable shifting of the strong visible absorption among the different rare gases. However, the dominance of the features in the visible region is entirely consistent with this band being due to the fully allowed $y^{8} \mathrm{P} \leftrightarrow a^{8} \mathrm{~S}$ electronic transition. The origins of the complex structures in the solid-state spectra will be analyzed in Secs. III B-III E.

\section{B. Dimer formation}

The Eu/RG absorption spectra presented in Fig. 2 following deposition at $10 \mathrm{~K}$ and recorded after sample annealing, 


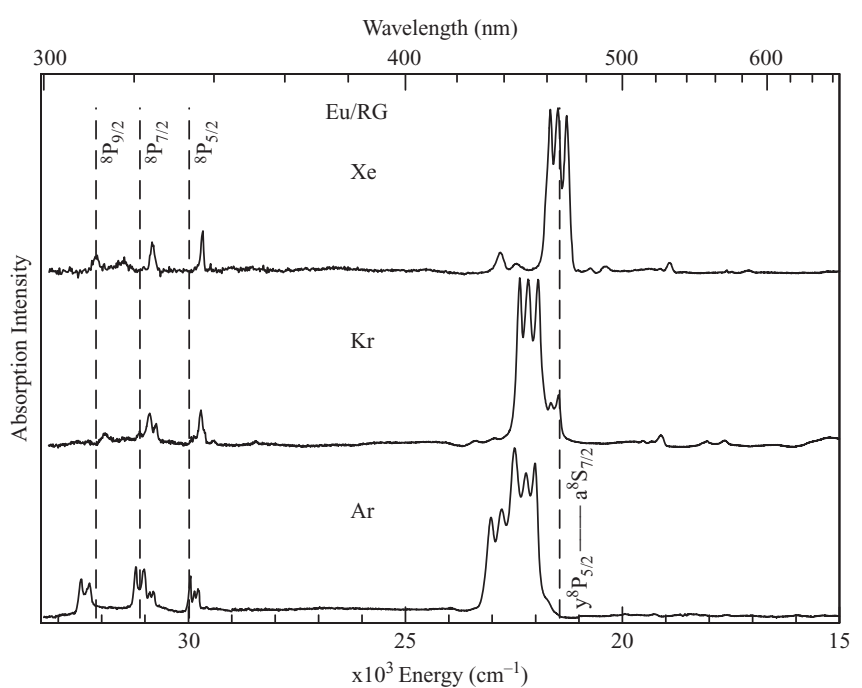

FIG. 2. UV-Vis absorption spectra recorded at $10 \mathrm{~K}$ following annealing of the Eu/RG samples. The gas phase position of the $y^{8} \mathrm{P}_{5 / 2} \leftarrow a^{8} \mathrm{~S}_{7 / 2}$ transition and transitions to the three spin-orbit levels of the ${ }^{8} \mathrm{P}_{\mathrm{J}}$ state are shown by the dashed vertical lines.

appear to exhibit almost complete atomic isolation even under high metal loading conditions. This is surprising, as the previous metal systems studied by us ( $\left.\mathrm{Ca}[\mathrm{Ar}] 4 \mathrm{~s}^{2}, \mathrm{Mn}[\mathrm{Ar}] 4 \mathrm{~s}^{2} 3 \mathrm{~d}^{5}\right)$ readily formed van der Waals metal dimers even with depositions at low temperature. It is known that the deposition temperature strongly affects the yield of atoms as compared to metal clusters. Only at very low temperatures it is possible to achieve high monomer yield, at higher temperatures aggregation is severe, even at very low metal concentrations. ${ }^{17}$ In an attempt to enhance the formation of Eu clusters, a concentration study was undertaken with higher deposition temperatures following the procedure outlined in Sec. II.

The bottom trace of Fig. 3 presents the absorption spectrum of $\mathrm{Eu} / \mathrm{Kr}$ recorded following deposition at $10 \mathrm{~K}$. Clearly evident is the $y^{8} \mathrm{P}$ state absorption centered at $460 \mathrm{~nm}$ but no other strong absorption features are identifiable. The remaining three traces in Fig. 3 display the effects of increased metal loading in samples deposited at $22 \mathrm{~K}$. With increased, but still low metal loading conditions, new weak absorption features are already observed in the $510 \mathrm{~nm}$ spectral region. These features at 498.8 (shoulder), 507.1 , and $519.6 \mathrm{~nm}$ become more prominent at medium and high metal loading, their corresponding growth at increased fluxes is indicative of metal aggregates. At the highest metal fluxes, additional absorption features are observed at 552.5, 567.0, and $579.6 \mathrm{~nm}$. The nonatomic absorption features observed in samples formed with low metal loading in the $510 \mathrm{~nm}$ region are attributed to the europium dimer, $\mathrm{Eu}_{2}$. The longer wavelength features which increase at higher metal loading are indicative of higher aggregates, i.e., $\mathrm{Eu}_{x}(x>2)$.

A comparison of the three Eu/RG systems deposited at elevated temperatures with high metal concentrations is presented in Fig. 4 and the spectral values are collected in Table I. The absorption spectra of samples deposited at $25 \mathrm{~K}$ in solid Xe yield a broad absorption feature centered at $513 \mathrm{~nm}$ which is observed to increase relative to the $y^{8} \mathrm{P}$ state atomic absorption at higher metal loading. This effect

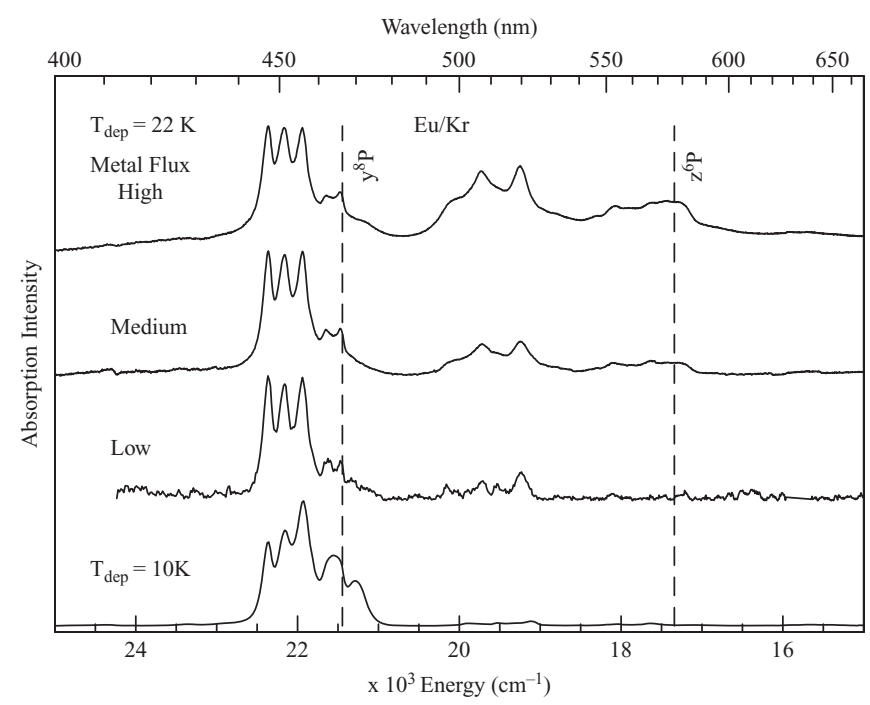

FIG. 3. Normalized Eu/Kr absorption spectra recorded at $10 \mathrm{~K}$. The spectrum recorded following sample deposition at $10 \mathrm{~K}$ with medium metal concentration is displayed in bottom trace. The remaining three spectra show the effects of increased metal flux, for depositions at $22 \mathrm{~K}$. The noise on the second lowest trace is due to weak absorption strength at low metal concentrations. The gas phase positions of the $y^{8} \mathrm{P}$ and $z^{6} \mathrm{P}$ states in this region are shown as dashed vertical lines.

is characteristic of a Eu dimer band. No other new absorption features are identified implying no higher aggregates of $\mathrm{Eu}_{x}(x>2)$ are formed in the Xe matrix under these conditions. This is reasonable considering $\mathrm{Xe}$ is the most polarizable of the three RG hosts; thus, increasing the van der Waals type interaction with the metal atom and thereby stabilizing their isolation within the lattice. In the case of Eu/Ar, two regions of the absorption spectrum show new features, a narrow doublet with peaks at 494.2 and $500.5 \mathrm{~nm}$ and a broader band with features at 545.7, 564.5 , and $579.9 \mathrm{~nm}$. With low metal loading the doublet at $500 \mathrm{~nm}$ is the dominant feature in the spectrum implying it must correspond to

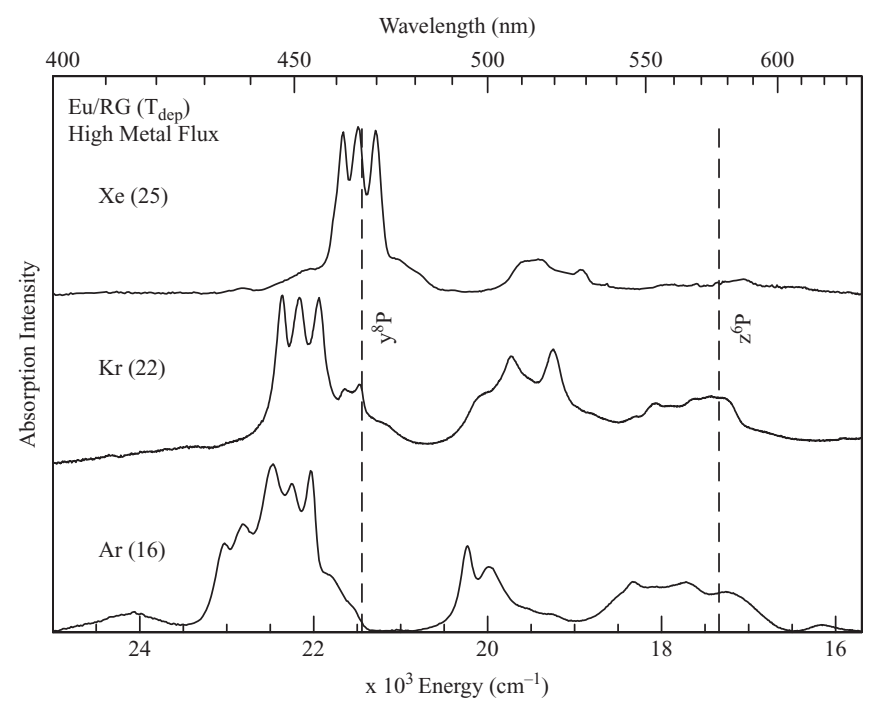

FIG. 4. Eu/RG visible absorption spectra recorded at $10 \mathrm{~K}$ following high temperature sample depositions with high metal fluxes. The gas phase position of the $y^{8} \mathrm{P}_{5 / 2}, z^{6} \mathrm{P}_{7 / 2} \leftarrow{ }^{8} \mathrm{~S}_{7 / 2}$ transitions are shown by the dashed vertical lines. 
TABLE I. The spectral location of europium dimer and higher aggregate absorption features $\left(\lambda_{\mathrm{Abs}}\right)$ in nanometer units for each of the three RG's (RG $=\mathrm{Ar}, \mathrm{Kr}$, and $\mathrm{Xe})$.

\begin{tabular}{llll}
\hline \hline & \multicolumn{3}{c}{$\mathrm{Eu} / \mathrm{RG}, \lambda_{\mathrm{Abs}}(\mathrm{nm})$} \\
\cline { 2 - 4 } Assignment & $\mathrm{Ar}$ & $\mathrm{Kr}$ & $\mathrm{Xe}$ \\
\hline & & $498.8(\mathrm{sh})$ & 510.7 \\
$\mathrm{Eu}_{2}$ & 494.2 & 507.1 & 515.3 \\
& 500.5 & 519.6 & 528.3 \\
& 545.7 & 552.5 & $\ldots$ \\
$\mathrm{Eu}_{x(x>2)}$ & 564.5 & 567 & $\ldots$ \\
& 580.5 & 579.9 & $\ldots$ \\
\hline \hline
\end{tabular}

europium dimer. Even though the bands were recorded at a resolution of $0.1 \mathrm{~nm}$ no vibrational fine structure was observed. In addition a careful search was made for cluster emission but none was observed out the long wavelength limit $(\lambda$ $=850 \mathrm{~nm}$ ) of our detection system.

\section{C. $E u\left(y^{8} P\right) / R G$ absorption/excitation spectroscopy}

Details of the Eu/RG visible absorption spectra are presented and compared in Fig. 5 for the three systems studied in the present work. Eu/Xe is the simplest host, consisting of three resolved absorption features at 462, 466, and $470 \mathrm{~nm}$ following the removal of a thermally unstable site by annealing. Excitation spectra recorded in this region, shown by the

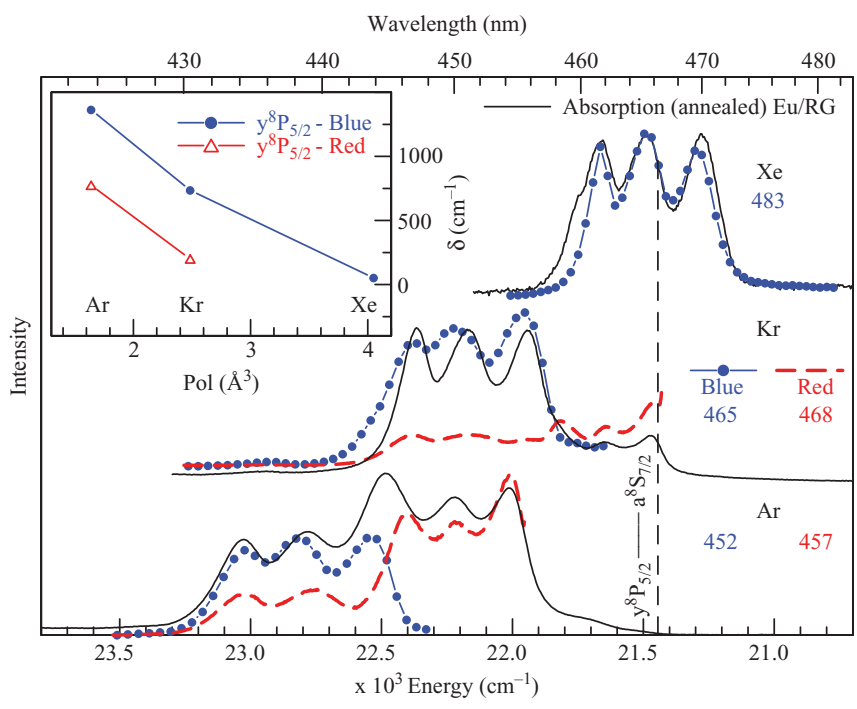

FIG. 5. Eu/RG absorption and site-specific excitation spectra recorded in the region of the $y^{8} \mathrm{P}_{5 / 2} \leftarrow{ }^{8} \mathrm{~S}_{7 / 2}$ transition at $10 \mathrm{~K}$ following sample annealing. The values provided on the right-hand side are the emission wavelengths used to record the excitation spectra. Two sites of isolation are identified, a higher energy blue site (dotted trace) and a lower energy red site (dashed trace). The inset plots the gas phase to matrix frequency shifts $\left(\delta \mathrm{cm}^{-1}\right)$ observed for the central Jahn-Teller component of the blue and red sites vs the RG host polarizabilities. The filled dots (connected by the solid line) highlight the linear correlation between the Eu $y^{8} \mathrm{P} \leftarrow a^{8} \mathrm{~S}$ transition occurring for the blue sites of isolation. Data on the red site is limited to just the $\mathrm{Ar}$ and $\mathrm{Kr}$ systems but exhibits the same slope but a much smaller absolute shift than the blue site.
TABLE II. Photophysical characteristics of the sites of isolation (red and blue) of $\mathrm{Eu} / \mathrm{RG}$ revealed in the excitation/absorption spectra of the $y^{8} \mathrm{P}$ $\left(4 \mathrm{f}^{7} 6 \mathrm{~s}^{1} 6 \mathrm{p}^{1}\right) \leftrightarrow a^{8} \mathrm{~S}_{7 / 2}\left(4 \mathrm{f}^{7} 6 \mathrm{~s}^{2}\right)$ transition of atomic europium. The dominant/primary $\left(1^{\circ}\right)$ and secondary $\left(2^{\circ}\right)$ sites are labeled to reflect their relative absorption strengths. The spectral position, $v$, and average linewidth (FWHM) of the three excitation components, $\Delta_{\mathrm{AV}}$, are quoted in wavenumber units. Gas phase to matrix frequency shifts are presented for the atomic Eu $y^{8} \mathrm{P}_{5 / 2} \leftarrow a^{8} \mathrm{~S}_{7 / 2}$ transition (G.P.: $21445 \mathrm{~cm}^{-1}$ ) as $\delta$ in wavenumber units. Noteworthy is the different magnitude of the threefold splittings present on the bands pertaining to the blue and red sites. This is most pronounced in $\mathrm{Kr}$ where values of 165 and $190 \mathrm{~cm}^{-1}$ are obtained for the red (differences of the entries in column 4) while larger values of 213 and $234 \mathrm{~cm}^{-1}$ exist for the blue.

\begin{tabular}{lccccc}
\hline \hline \multicolumn{5}{c}{ Eu $\left(y^{8} \mathrm{P}\right) / \mathrm{RG}$ excitation features } \\
\hline Eu/RG site & Component & $\lambda(\mathrm{nm})$ & $v\left(\mathrm{~cm}^{-1}\right)$ & $\Delta_{\mathrm{AV}}\left(\mathrm{cm}^{-1}\right)$ & $\delta\left(\mathrm{cm}^{-1}\right)$ \\
\hline Argon & 1 & 446.4 & 22401 & & 956 \\
Red $\left(1^{0}\right)$ & 2 & 450.1 & 22217 & $168^{\mathrm{a}}$ & 772 \\
& 3 & 454.4 & 22007 & & 562 \\
& & & & & \\
Blue $\left(2^{0}\right)$ & 2 & 438.4 & 22810 & 200 & 1396 \\
& 3 & 443.6 & 22543 & & 1098 \\
Krypton & 1 & 446.6 & 22391 & & 946 \\
Blue $\left(1^{0}\right)$ & 2 & 450.9 & 22178 & 208 & 733 \\
& 3 & 455.5 & 21954 & & 509 \\
& & & & & 365 \\
Red $\left(2^{0}\right)$ & 2 & 458.5 & 21810 & & 200 \\
Xenon & 3 & 462.0 & 21645 & $88^{\mathrm{a}}$ & 10 \\
$\left(1^{0}\right)$ & 1 & 466.1 & 21455 & & 223 \\
\hline \hline & 2 & 465.2 & 21496 & 137 & 51 \\
& 3 & 469.6 & 21295 & & -150 \\
\hline
\end{tabular}

a Represents $\Delta_{\mathrm{AV}}$ was calculated using only two JT components as the third one was not fully resolved.

dotted line in the top panel, are consistent with the absorption spectrum, confirming the existence of only a single thermally stable site of isolation for Eu atoms in Xe matrices. The Eu/Xe $y^{8} \mathrm{P}$ state excitation components have an average linewidth (FWHM) of $137 \mathrm{~cm}^{-1}$ and exhibit a small blue matrix shift of $51 \mathrm{~cm}^{-1}$ from the gas phase $y^{8} \mathrm{P}_{5 / 2} \leftarrow a^{8} \mathrm{~S}_{7 / 2}$ atomic transition. The quoted matrix shift is calculated from the central Jahn-Teller component and the gas phase $J=5 / 2$ spin-orbit level. The results obtained in this way are listed in Table II.

Annealing the Eu/Ar matrix to $29 \mathrm{~K}$ produced a structured absorption band centered at $445 \mathrm{~nm}$ (shown by the solid trace on the bottom in Fig. 5) exhibiting five resolved features at $434.3,439.0,444.8,450.1$, and $454.3 \mathrm{~nm}$. These five absorption features suggest the existence of two thermally stable sites for the isolation of atomic Eu in Ar in roughly comparable amounts. Excitation spectra recorded in this region monitoring site-specific emission features show two well resolved bands arising from two distinct sites of isolation. Both bands exhibit threefold splitting indicating the occurrence of Jahn-Teller coupling. The spectral characteristics of the excitation features of each site are collected in Table II. The three excitation components of the blue site exhibit an average width (FWHM) of $200 \mathrm{~cm}^{-1}$ and a blue matrix shift from the gas phase atomic transition of $1364 \mathrm{~cm}^{-1}$. The red site 
excitation features yield a smaller average width of $168 \mathrm{~cm}^{-1}$ and a smaller matrix blue shift of $774 \mathrm{~cm}^{-1}$.

The absorption spectrum recorded for an annealed $\mathrm{Eu} / \mathrm{Kr}$ sample, displayed in the middle trace of Fig 5, suggests the presence of two thermally stable sites of isolation for $\mathrm{Eu}$ atoms in $\mathrm{Kr}$ matrices. In contrast to $\mathrm{Eu} / \mathrm{Ar}$, the quantities of the two sites are now quite unequal. Nevertheless, siteselective excitation spectra recorded in the vicinity of the $y^{8} \mathrm{P}$ $\leftarrow a^{8} \mathrm{~S}$ transition allow clear identification of two distinct europium atom trapping sites in solid $\mathrm{Kr}$. A dominant blue site exhibiting characteristic Jahn-Teller threefold splitting features at $446.6,450.9$, and $455.5 \mathrm{~nm}$ and a weaker red site with features centered at $458.5,462.0$, and $466.1 \mathrm{~nm}$. The red site is more than five times weaker than the blue site. The characteristics of the three components in each excitation spectrum are provided in Table II. The blue site excitation components have an average width of $208 \mathrm{~cm}^{-1}$ and matrix shift of $735 \mathrm{~cm}^{-1}$. Europium atoms isolated in the red site exhibit a much smaller bandwidth of $88 \mathrm{~cm}^{-1}$ and a blue shift of only $200 \mathrm{~cm}^{-1}$.

\section{D. $\operatorname{Eu}\left(z^{6} P\right) / R G$ excitation spectroscopy}

Excitation spectra recorded in the region of the $z^{6} \mathrm{P}$ state for the blue site in each rare gas host are displayed in the left hand panel of Fig. 6. In solid Ar two structureless features centered at 541 and $552.7 \mathrm{~nm}$ are observed while in $\mathrm{Kr}$, they are centered at 554.2 and $567 \mathrm{~nm}$. In Eu/ Kr the lower energy excitation component is structureless whilst the higher energy band exhibits two identifiable features. This $554.2 \mathrm{~nm}$ band is fully accounted for by two Gaussian curves of widths 126 and $89 \mathrm{~cm}^{-1}$ centered at 551.1 and $554.2 \mathrm{~nm}$, respectively. Excitation spectra recorded in solid Xe present two in-

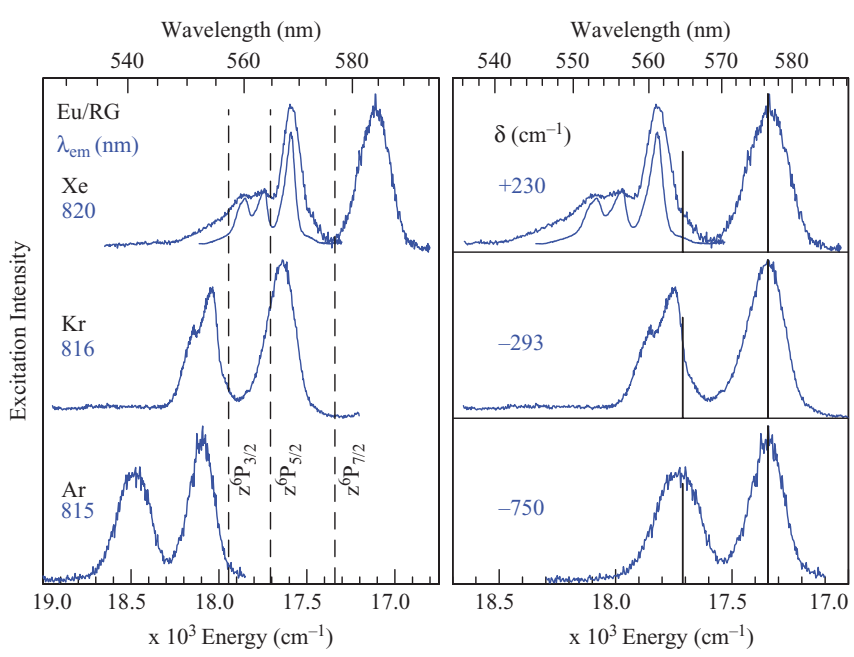

FIG. 6. Eu/RG blue site excitation spectra recorded in the region of the $z^{6} \mathrm{P}$ state of atomic Eu are shown in the panel on the left-hand side. The emission wavelengths used to record the excitation spectra are indicated as the $\lambda_{\mathrm{em}}$ values. The spectral positions of each of the three $z^{6} \mathrm{P}$ spin-orbit levels in the gas phase are shown by the dashed vertical lines. In the panel on the right-hand side the recorded spectra are shifted by the amount indicated by $\delta\left(\mathrm{cm}^{-1}\right)$ to align with the gas phase $z^{6} \mathrm{P}_{7 / 2}$ level. The relative intensities of the two observed gas phase transitions ${ }^{32}(J=5 / 2,7 / 2)$ are shown by the stick spectra. tense features at 568.7 and $584.4 \mathrm{~nm}$. The feature centered at $584.4 \mathrm{~nm}$ is structureless while the band at $568.7 \mathrm{~nm}$ suggests some further structure to higher energy. A high-resolution laser excitation scan of this band is displayed in the upper portion of Fig. 6 which allows identification of three constituent components.

In the right-hand panel of Fig. 6 the lowest energy components of the $\mathrm{Eu} / \mathrm{RG}$ excitation spectra have been shifted by the indicated values, to coincide with the gas phase $z^{6} \mathrm{P}_{7 / 2}-$ $a^{8} \mathrm{~S}_{7 / 2}$ transition. When the observed excitation spectrum in Ar is shifted by $-750 \mathrm{~cm}^{-1}$ there is almost perfect agreement between the spectral location of the experimental data and the gas phase positions of the $J=5 / 2,7 / 2$ levels of the $z^{6} \mathrm{P}$ state. The stick spectra ${ }^{18}$ shown in this panel, indicate the relative intensities of the two transitions allowed in the gas phase, ${ }^{19}$ revealing a very close correspondence with the matrix spectra.

There is also good agreement between the experimental excitation spectra of $\mathrm{Eu} / \mathrm{Kr}$ and $\mathrm{Eu} / \mathrm{Xe}$ and the gas phase transitions of $z^{6} \mathrm{P}_{\mathrm{J}}(J=5 / 2,7 / 2)$ when matrix shifts of -293 and $+230 \mathrm{~cm}^{-1}$ are accounted for. The very close correspondence between the matrix and the gas phase spectra strongly suggests that two of the three spin-orbit levels of the $z^{6} \mathrm{P}_{\mathrm{J}}$ state are present in the matrix excitation spectra. All the bands shown in Fig. 6 are excitation spectra recorded by monitoring the specified emission wavelengths which correspond to transitions of the metastable ${ }^{10} \mathrm{D}$ state of the atom. Thus all these bands must be atomic in origin. While Eu dimers and higher clusters do absorb in this spectral region, such species were absent in the samples used to record the excitation spectra shown in Fig. 6. It is thus proposed that the two excitation features observed in each RG correspond to the $J=5 / 2,7 / 2$ levels of the $z^{6} \mathrm{P}$ state. This is based on both the similar splitting and intensities observed in the RG hosts and what is observed for these levels in the gas phase. A very interesting question arises from the above assignment, viz., why do JT forces dominate in the resonance $y^{8} \mathrm{P}$ transition and not in the lower energy $z^{6} \mathrm{P}$ state? While a definitive answer is currently not at hand, it is noteworthy that the spin-orbit splitting of the $z^{6} \mathrm{P}$ state $\left(\zeta=604.8 \mathrm{~cm}^{-1}\right)$ is roughly twice that of the $y^{8} \mathrm{P}$ state $\left(\zeta=316.7 \mathrm{~cm}^{-1}\right)$. Also, as the strength of interaction of the guest and the lattice increases from Ar to Xe, the extent of JT stabilization increases. This is consistent with the observation of a threefold split $J=5 / 2$ level for the $z^{6} \mathrm{P}$ state in $\mathrm{Eu} / \mathrm{Xe}$.

\section{E. Near-UV Eu $\left({ }^{8} \mathrm{P}\right) / \mathrm{RG}$ excitation}

Steady-state excitation spectra recorded herein for the $300-340 \mathrm{~nm}$ region, monitoring the site-specific $y^{8} \mathrm{P}$ state fluorescence $(\sim 450 \mathrm{~nm})$ in annealed samples, allowing distinction of these narrow features previously reported in the higher resolution data of Jakob et al. ${ }^{12}$ to be made on the basis of the red $(\mathrm{R})$ and blue (B) site occupancy. The site-selective excitation spectra are presented in Fig. 7 for the three rare gases studied. The state assignments given by Jakob et al. for these spectral features as absorptions of the individual $J$-levels of the ${ }^{8} \mathrm{P}_{\mathrm{J}}(J=5 / 2,7 / 2,9 / 2)$ and ${ }^{6} \mathrm{P}_{\mathrm{J}}(J=5 / 2$ and $7 / 2)$ states 


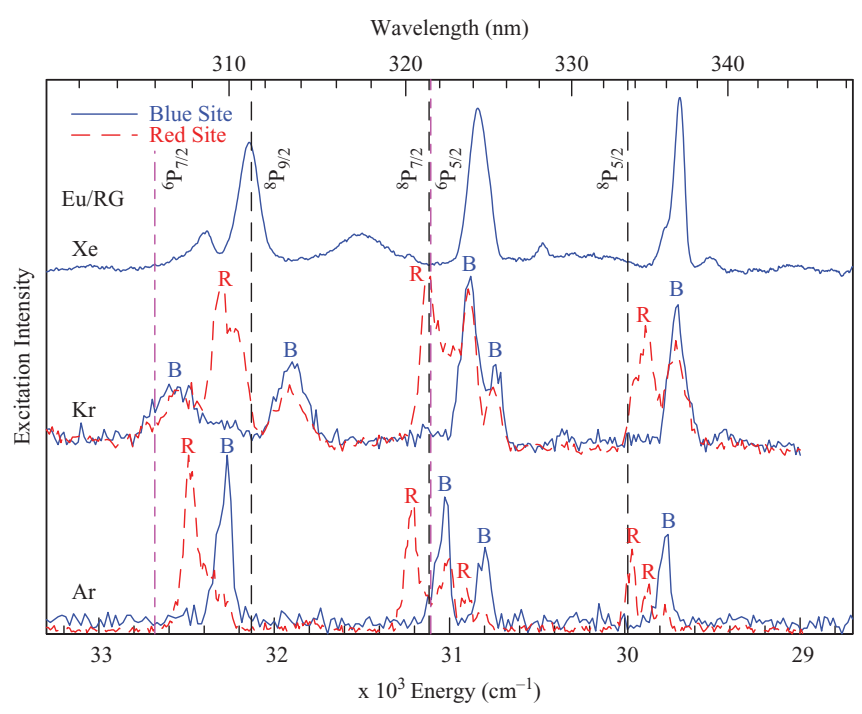

FIG. 7. Eu/RG site-specific excitation spectra in the near-UV spectral region recorded at $10 \mathrm{~K}$ following sample annealing. Two sites of isolation are identified, the blue site ( $\mathrm{B}$, solid trace) and the red site ( $\mathrm{R}$, dashed trace). The gas phase positions of the ${ }^{8} \mathrm{P}_{\mathrm{J}},{ }^{6} \mathrm{P}_{\mathrm{J}} \leftarrow a^{8} \mathrm{~S}_{7 / 2}$ transitions are identified by the dashed vertical lines.

are correct. However, the origin of the underlying structure is more complicated than CF splitting alone as multiple site occupancy in the solid Ar and $\mathrm{Kr}$ lattices is clearly present as depicted in the excitation spectra of Fig. 7. The presence of multiple site occupancy in $\mathrm{Ar}$ and $\mathrm{Kr}$ can also be inferred from the relative simplicity of the $\mathrm{Eu} / \mathrm{Xe}$ spectra. Absorption features can now be attributed to atomic Eu occupying a particular site of isolation. The nature and symmetry of these sites will be discussed in Sec. IV B.

\section{DISCUSSION}

\section{A. Dimer formation}

It appears that the matrix samples formed at $10 \mathrm{~K}$ contain very small amounts of Eu clusters. Aggregates are only observed when specific conditions, favorable for cluster formation, were deployed such as the use of higher deposition temperatures. This behavior is consistent with that previously observed by Suzer and Andrews ${ }^{14}$ on ytterbium dimer. It is not entirely surprising as Eu, with the $4 f^{7} 6 s^{2}$ valence electron configuration, has an outer closed s-orbital which determines that the dimer formed will be a van der Waals type molecule with a weak bond and a long bond length. This expectation is supported by calculations by Cao and Dolg. ${ }^{20}$ who predict a high spin $\left(4 \mathrm{f}^{7} 4 \mathrm{f}^{7} \sigma_{\mathrm{g}}{ }^{2} \sigma_{\mathrm{u}}{ }^{215} \Sigma^{+}{ }_{\mathrm{u}}\right)$ ground state for europium dimer with a bond length of $4.878 \AA$ and a binding energy of only $0.08 \mathrm{eV}\left(645 \mathrm{~cm}^{-1}\right)$. Buchachenko proposes ${ }^{21}$ a low spin $\left({ }^{1} \Sigma^{+} \mathrm{g}\right)$ ground state but with a similarly weak binding energy of $710 \mathrm{~cm}^{-1}$.

Europium is a heavy metal atom (152 amu) and selfaggregated to a much lesser extent in the matrix than other van der Waals metal dimers studied by the Maynooth group such as manganese. ${ }^{6}$ The difference may be related to the larger mass of this guest, which is three times heavier than any metal atom previously studied. Due to their increased mass,
$\mathrm{Eu}$ atoms will have less kinetic energy in the growing surface layer after efficient momentum transfer to the solid upon collision at the sample. This reduced mobility will limit the chances of encountering another europium atom, thereby decreasing the probability of dimer formation at $10 \mathrm{~K}$. Furthermore, the predicted binding energies of the Eu-RG diatomics are, as shown in Table III, consistently larger than those of the rare gas dimers, suggesting that the host atoms will bind preferentially with the guest atom. However, as also shown in Table III, the Eu-RG bond lengths are much larger than those of the RG-RG dimers making occupancy in single vacancy sites improbable.

\section{B. Site occupancy}

The recorded excitation spectra allow resolution of the congested $\mathrm{Eu} / \mathrm{Ar}$ and $\mathrm{Eu} / \mathrm{Kr}$ absorption spectra into distinct sites of isolation for atomic Eu in these rare gases. The excitation spectra reveal the presence of threefold split patterns for each of the sites which is attributed to the Jahn-Teller effect. This is indicative of Eu atoms occupying highly symmetric matrix environments.

Based on the expectation that the excited $\mathrm{P}$ state is increasingly stabilized by the heavier rare gases, a trend to lower absorption energy is predicted in the more polarizable RG hosts. Laursen and Cartland ${ }^{22}$ showed for group XII metal atoms $(\mathrm{Hg}, \mathrm{Cd}$, and $\mathrm{Zn}$ ) that the matrix shift for atomic $\mathrm{P} \leftarrow$ $\mathrm{S}$ transitions is approximately linear when plotted against the polarizability of the RG's. This behavior was also found ${ }^{3}$ in the case of matrix-isolated atomic Mn. The observed matrix shift ${ }^{23}$ from the gas phase $y^{8} \mathrm{P}_{5 / 2} \leftarrow a^{8} \mathrm{~S}_{7 / 2}$ transition of atomic $\mathrm{Eu}$ is plotted against the RG polarizability in the inset of Fig. 5. The linear correlation between the single thermally stable site of isolation in $\mathrm{Xe}$ and the blue sites in $\mathrm{Ar}$ and $\mathrm{Kr}$ suggests atomic Eu is trapped in the same type of site for the blue sites present in $\mathrm{Ar}$ and $\mathrm{Kr}$ and the one site occupied in $\mathrm{Xe}$. The red sites in the $\mathrm{Ar}$ and $\mathrm{Kr}$ systems appear to show the same slope as the three blue sites present in the three rare gases studied, but with a much smaller absolute shift from the gas phase.

To the best of our knowledge no bond length data are available either experimentally or from $a b$ initio calculations for the Eu-RG diatomics. In the absence of such data, estimates of the Eu-RG diatomic bond lengths can be obtained using the Luiti-Pirani ${ }^{24}$ (LP) method. This is a simple approach, relying only on the atomic ionization potential $(I)$ and polarizability $(\alpha)$, to determine approximate expressions for the bond lengths $\left(R_{e}\right)$ and dissociation energies $\left(D_{e}\right)$ of metal-RG van der Waals diatomics. To evaluate the accuracy of this method, sodium was chosen as a test system since its polarizability and ionization potential are similar to atomic $\mathrm{Eu}$. The values used are listed in the caption of Table III. More significantly the Na-RG diatomic bond lengths are now known experimentally from very accurate, high-resolution spectroscopy by Zimmermann and co-workers. ${ }^{25,26}$ Finally, since $\mathrm{Na}$ and Eu exist in spherical ground electronic states, ${ }^{2} \mathrm{~S}$ and ${ }^{8} \mathrm{~S}$, respectively, both of these atoms will show a preference for spherical site occupancy in the $f c c$ lattice structure of the solid RG's. 
TABLE III. A comparison of the known (exp) Na-RG diatomic bond lengths and values predicted using the Luiti-Pirani (LP) technique. The percentage difference between both is given and less than $4 \%$ in all cases (columns 1-4). The Eu-RG diatomic bond lengths calculated with the LP technique. In the LP calculations, values of 24.1 and $27.7 \AA^{3}$ were used for the ground state polarizability $(\alpha)$ of atomic Na and Eu, respectively, while ionization potentials of 5.14 and $5.67 \mathrm{eV}$ were used (columns 5-7). These values were obtained from CRC Handbook of Chemistry and Physics, 76th ed. (CRC, Boca Raton, 1995). The LP predictions for the rare gas dimers are provided in columns 8-10.

\begin{tabular}{cccccccccc}
\hline \hline $\mathrm{Na}$ & $R_{e}(\AA) \exp$ & $R_{e}(\AA) \mathrm{LP}$ & $(\%)$ & $\mathrm{Eu}$ & $R_{e}(\AA) \mathrm{LP}$ & $D_{e}\left(\mathrm{~cm}^{-1}\right) \mathrm{LP}$ & $\mathrm{RG}$ & $R_{e}(\AA)$ & $D_{e}\left(\mathrm{~cm}^{-1}\right)$ \\
\hline $\mathrm{Ar}$ & 5.01 & 5.07 & 1.2 & $\mathrm{Ar}$ & 5.17 & 86.4 & $\mathrm{Ar}$ & 3.755 & 61.98 \\
$\mathrm{Kr}$ & 4.92 & 5.08 & 3.3 & $\mathrm{Kr}$ & 5.18 & 125.6 & $\mathrm{Kr}$ & 3.988 & 88.3 \\
$\mathrm{Xe}$ & 4.95 & 5.13 & 3.6 & $\mathrm{Xe}$ & 5.21 & 187.9 & $\mathrm{Xe}$ & 4.330 & 133.4 \\
\hline \hline
\end{tabular}

When the experimental $\mathrm{Na}-\mathrm{RG}$ bond lengths are compared to the results predicted with the LP method, good agreement is achieved. Only small differences in the range of $1 \%$ 4\% are observed as shown in Table III. This supports the expectation that the LP method can provide a reliable estimate of the bond length for an S-state metal atom-RG van der Waals diatomic. The Eu-RG diatomic bond lengths were then estimated by the LP technique, the results of which are presented in Table III. The Eu-RG bond lengths predicted by the LP method are of the same order of magnitude as $\mathrm{Na}-\mathrm{RG}$, but are approximately $0.1 \AA$ longer than the sodium diatomics in all cases. The comparable diatomic bond lengths suggest Eu will exhibit similar trapping site preferences as atomic $\mathrm{Na}$ in the RG matrices.

A recent molecular dynamics study ${ }^{27}$ of matrix-isolated atomic ${ }^{5}$ sodium attributes the red and blue sites to hexavacancy (hv) and tetra-vacancy (tv) site occupancy, respectively, in $\mathrm{Ar}$ and $\mathrm{Kr}$ matrices. ${ }^{28}$ The tv site is created by the removal of the four adjacent host atoms (arranged as a tetrahedron) surrounding the tetrahedral interstitial $\left(I_{\mathrm{Td}}\right)$ site in the $f c c$ lattice. When the six nearest neighbor atoms, arranged as an octahedron, surrounding an octahedral interstitial $\left(I_{\mathrm{oh}}\right)$ site are removed, the hv site is generated. Sodium shows a preference for the hv site in solid Ar; thus, Eu would be expected to show similar behavior allowing the dominant red site of isolation of atomic Eu in Ar to be attributed to a hv site. Sodium displays a slight preference for tv site occupancy (56\%) in solid $\mathrm{Kr}$, suggesting the dominant site of isolation of $\mathrm{Eu}$ in the $\mathrm{Kr}$ lattice, the blue site, relates to a tv. The Eu/RG polarizability plot shown in Fig. 5 allows association of the blue sites of isolation in $\mathrm{Ar}$ and $\mathrm{Kr}$ as the same site type as the single site present in Xe which is attributed to a tetra-vacancy site. The sizes of the available spherical vacancies in the $f c c$ RG lattices along with the LP predicted Eu-RG diatomic bond lengths are listed in Table IV for comparison purposes.

TABLE IV. Site sizes (in $\AA$ ) for specific spherically symmetric site types in the solid rare gases. Also shown is the lattice parameter, $a$, of each $f_{c c}$ solid. In addition, the LP calculated $\mathrm{Eu}\left({ }^{8} \mathrm{~S}_{7 / 2}\right)-\mathrm{RG}$ diatomic ground state bond lengths are presented.

\begin{tabular}{lcccccc}
\hline \hline $\mathrm{RG}$ & $a(\AA)^{\mathrm{a}}$ & $I_{\text {oh }}(\AA)$ & $\mathrm{sv}(\AA)$ & tv $(\AA)$ & hv $(\AA)$ & $\operatorname{Eu}-\mathrm{RG}(\AA)$ \\
\hline $\mathrm{Ar}$ & 5.31 & 2.655 & 3.755 & 4.403 & 4.598 & 5.17 \\
$\mathrm{Kr}$ & 5.64 & 2.822 & 3.988 & 4.676 & 4.884 & 5.18 \\
$\mathrm{Xe}$ & 6.13 & 3.065 & 4.330 & 5.083 & 5.308 & 5.21 \\
\hline \hline
\end{tabular}

a Reference 33 .

\section{Near-UV Eu( $\left({ }^{8} \mathrm{P}\right) / \mathrm{Ar}$ reanalyzed}

High-resolution absorption spectra of the near-UV ${ }^{8} \mathrm{P}_{5 / 2}$ state were previously recorded by Jakob et al. ${ }^{12}$ for $\mathrm{Eu}$ in Ar matrices and these data are compared with the current site-selective excitation spectra in Fig. 8. Temperature dependent studies of the high-resolution absorption spectra identified three clear zero phonon lines (ZPL's), each with a highenergy phonon sideband. It was assumed previously that the $\mathrm{Eu}$ atom existed in a single site in the Ar lattice and the ZPL's were proposed as arising from individual weak field states of the $J=5 / 2$ level split by a noncubic crystal field. However, comparison with the current site-specific Eu/Ar excitation data allows the absorption features to be attributed to distinct sites.

The effect of the cubic CF on the Eu atom ${ }^{8} \mathrm{P}_{5 / 2}$ state in the hv and tv lattice sites is predicted, by Group theoretical methods, ${ }^{29}$ to split the $J=5 / 2$ spin-orbit level into two weak field components labeled $E_{3}$ and $G$, having degeneracies of 2 and 4 , respectively. Thus, in a field of cubic symmetry the spin-orbit $J=5 / 2$ level will split into two components with relative intensities of $2: 1$. To examine this prediction a detailed analysis of the resolved bands was conducted with the Wp lineshape function. ${ }^{30}$ With a phonon frequency, $h \omega$, of $27 \mathrm{~cm}^{-1}$ (a value selected from the resolved phonon sidebands) and the experimental temperature of $4 \mathrm{~K}$, the only adjustable parameter in the $\mathrm{Wp}$ function fit is the Huang-Rhys factor $(S)$. Using an $S$ value of 0.35 the simulations presented on the upper right of Fig. 8 were obtained. As shown the red site of isolation presents two ZPL's of intensity 2:1 located at 333.7 and $334.9 \mathrm{~nm}$. Similarly, two ZPL's of weaker intensity are identifiable at 336.1 and $334.7 \mathrm{~nm}$ for the blue site. The occurrence of additional structure on the dominant CF bands of both sites (336.1 and $333.7 \mathrm{~nm}$ ) can be attributed to resolved phonon structure. From the locations of the pair of ZPL's of each site, the magnitude of the crystal field splittings on the $J=5 / 2$ level in Ar are found to be 105 and $123 \mathrm{~cm}^{-1}$ for the red and blue sites, respectively.

The absorption features in the 322 and $309 \mathrm{~nm}$ regions contain much more structure but the bands here are complicated by the fact that two distinct $\mathrm{P}$ states occur in this region. Thus in the $322 \mathrm{~nm}$ spectral region transitions to both the ${ }^{8} \mathrm{P}_{7 / 2}$ and ${ }^{6} \mathrm{P}_{5 / 2}$ states occur while in the $310 \mathrm{~nm}$ region, the ${ }^{8} \mathrm{P}_{9 / 2}$ and ${ }^{6} \mathrm{P}_{7 / 2}$ states absorb. Without the temperature dependence study performed at high resolution over this whole spectral region, it is not possible to accurately assign which features are due to ZPL's and which are sideband structures. Notwithstanding 

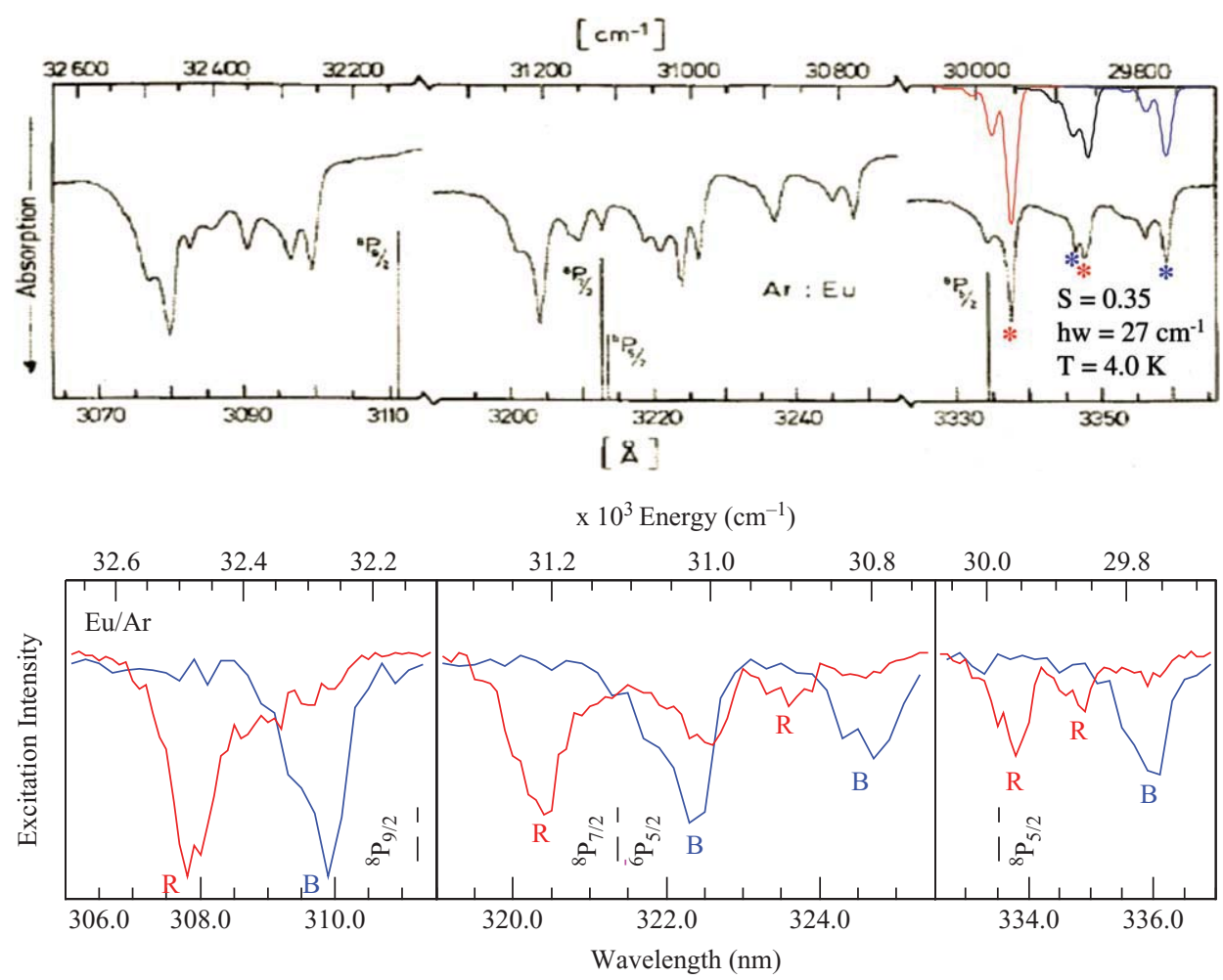

FIG. 8. The top panel presents absorption spectra of an annealed Eu/Ar sample recorded in the earlier study by Jakob et al. while the excitation data shown in the lower panel is from the present work. The comparison reveals the absorption spectrum in Eu/Ar is made up of a complex combination of site structure and crystal field splitting. The Wp lineshape analysis of the high-resolution ${ }^{8} \mathrm{P}_{5 / 2}$ absorption, based on the existence of a pair of thermally stable sites for Eu/Ar, was conducted with a single variable S, yielding a Huang-Rhys factor of 0.35 indicating weak electron-phonon coupling. The experimental temperature of 4 $\mathrm{K}$ was used and the phonon frequency of $27 \mathrm{~cm}^{-1}$ was taken from the resolved phonon sidebands. Each site exhibits a pair of zero phonon lines (marked by asterisks), those of the red site (R) are split by $105 \mathrm{~cm}^{-1}$ while the splitting on the blue site (B) is $123 \mathrm{~cm}^{-1}$. These values are identified as the magnitudes of the crystal splitting of the Eu atom $J=5 / 2$ level in the larger hexa-vacancy and the smaller tetra-vacancy sites, respectively. In generating the Wp lineshapes a bandwidth of $7 \mathrm{~cm}^{-1}$ was used to simulate the instrument resolution. Reprinted with permission from M. Jakob, H. Micklitz, and K. Luchner, Phys. Lett A 57, 67 (1976). Copyright 1976, Elsevier.

this, it is possible using the present excitation data to develop the previous work of Jakob et al. ${ }^{12}$ and attribute specific features in each of the RG matrices to atomic Eu isolated in a particular site. Site-specific features are labeled red and blue sites ( $\mathrm{R}$ and $\mathrm{B}$ ) in Fig. 7 on the basis of their location on the lower energy $y^{8} \mathrm{P}$ state excitation spectra. It is noteworthy that the blue site features occur to lower energy than the red site for the $\mathrm{f} \rightarrow \mathrm{d}$ type transitions in the near-UV region. This is an energy reversal compared to the $y^{8} \mathrm{P}$ state indicating very different excited state interactions for the $\mathrm{Eu}$ atom in $\mathrm{d}$ - and p-type excited states.

\section{CONCLUSIONS}

Europium atoms were isolated easily in RG matrices, while specific conditions involving sample formation at elevated temperatures, were required to form metal aggregates. This behavior is attributed to the large mass of the guest metal atom and the weak metal dimer van der Waals bond, both of which reduce the probability of metal aggregation. A strong similarity was observed between the visible absorption spectra of the Eu matrix systems and the Na systems both of which exhibit a pair of sites in $\mathrm{Eu} / \mathrm{Ar}$ and $\mathrm{Eu} / \mathrm{Kr}$ samples but only a single thermally stable site in Xe. This behavior can be traced back to the very similar ground state van der Waals bond lengths present in the 1:1 complexes, these metal atoms form with the rare gas atoms. Drawing from the results of the previous in-depth molecular dynamics study ${ }^{5}$ of matrix-isolated atomic sodium, tentative site assignments are provided for the $\mathrm{Eu} / \mathrm{RG}$ systems. The site types occupied by Eu atoms within the matrices are proposed as a tv for the blue site and a hv for the red site.

The absorption features observed in the near-UV spectral region relating to $\mathrm{f} \rightarrow \mathrm{d}$ type transitions are in agreement with an earlier study. Recording site-specific excitation spectra has allowed us to identify absorption features pertaining to $\mathrm{Eu}$ atoms isolated in different sites within the RG lattices. The threefold splitting pattern present on the $y^{8} \mathrm{P}$ state transition in the visible for these sites indicates that both are of cubic symmetry. The excited state behavior of matrix-isolated atomic $\mathrm{Eu}$ is quite varied. It spans the extremes where pure atomic spin-orbit splittings are maintained on the $\mathrm{f} \rightarrow \mathrm{d}$ type transitions in the near-UV, to where Jahn-Teller coupling ${ }^{31}$ wipes out such splitting on the $\mathrm{s} \rightarrow \mathrm{p}$ type $y^{8} \mathrm{P}$ transitions in the visible. The lower energy $z^{6} \mathrm{P}$ state exhibits both of these extreme behaviors depending on the host used to accommodate the Eu atom. Excitation spectra in the region of the $z^{6} \mathrm{P}$ state of atomic Eu isolated in the solid rare gases in the blue site of isolation allow identification of the $J=5 / 2$ and $7 / 2$ spin-orbit levels in the inert hosts. Interestingly, the $J=7 / 2$ excitation 
feature is structureless in all hosts while the $J=5 / 2$ level exhibits some splitting, the extent of which increases in larger, more polarizable hosts.

\section{ACKNOWLEDGMENTS}

This research was funded by the Embark Initiative Irish Research Council for Science and Engineering (IRCSET) and a John \& Pat Hume scholarship from N.U.I.-Maynooth to whom O.B. gratefully acknowledges receipt of a $\mathrm{Ph} . \mathrm{D}$. studentship. The iCCD camera used in this work was acquired with the financial support of Science Foundation Ireland (SFI), Investigator Grant No.02/IN.1/B032.

${ }^{1}$ B. Healy and J. G. McCaffrey, J. Chem. Phys. 110(8), 3903 (1999).

${ }^{2}$ V. A. Bracken, P. Gurtler, and J. G. McCaffrey, J. Chem. Phys. 107(14), 5290 (1997)

${ }^{3}$ M. A. Collier and J. G. McCaffrey, J. Chem. Phys. 119, 11878 (2003).

${ }^{4}$ P. Kerins, B. Healy, and J. G. McCaffrey, Low Temp. Phys. 26(9), 756 (2000); J. G. McCaffrey and G. A. Ozin, J. Chem. Phys. 101(12), 10354 (1994).

${ }^{5}$ M. Ryan, M. Collier, P. de Pujo, C. Crepin, and J. G. McCaffrey, J. Phys. Chem. A 114(9), 3011 (2009).

${ }^{6}$ M. Collier, Ph.D. dissertation, National University of Ireland Maynooth, 2004.

${ }^{7}$ M. A. Collier, O. Byrne, C. Murray, and J. G. McCaffrey, J. Chem. Phys. 132(16), 164512 (2010).

${ }^{8}$ M. A. Collier and J. G. McCaffrey, J. Chem. Phys. 122(5), 054503 (2005); 122(18), 184507 (2005); M. A. Collier, M. C. Ryan, and J. G. McCaffrey, J. Chem. Phys. 123(4), 044508 (2005).

${ }^{9}$ M. Rasanen, L. A. Heimbrook, and V. E. Bondybey, J. Mol. Struct. 157, 129 (1986).

${ }^{10}$ A. I. Krylov, R. B. Gerber, and V. A. Apkarian, Chem. Phys. 189, 261 (1994).

${ }^{11}$ A. A. Belyaeva and Y. B. Predtechenskii, Opt. Spectrosc. 60(6), 700 (1986).

${ }^{12}$ M. Jakob, H. Micklitz, and K. Luchner, Berichte Der Bunsen-Gesellschaft: Phys. Chem. Chem. Phys. 82(1), 32 (1978).

${ }^{13}$ M. Jakob, H. Micklitz, and K. Luchner, Phys. Lett. A 61(4), 265 (1977); 57(1), 67 (1976).

${ }^{14}$ S. Suzer and L. Andrews, J. Chem. Phys. 89(9), 5514 (1988).
${ }^{15}$ W. E. Klotzbucher, M. A. Petrukhina, and G. B. Sergeev, J. Phys. Chem. A 101(25), 4548 (1997).

${ }^{16}$ NIST Atomic Spectra Database (version 3.1.5), August 2010 (National Institute of Standards and Technology, Gaithersburg, MD). Available: http://physics.nist.gov/asd3.

${ }^{17}$ Y. Haas and U. Samuni, Prog. React. Kinet. Mech. 23(4), 211 (1998).

${ }^{18}$ Atomic Eu exists in the $J=7 / 2$ level of the a8S ground state so that transitions to the $J=5 / 2$ and $7 / 2$ levels of the z6PJ state are allowed corresponding to $\Delta J=-1$ and 0 , respectively. Excitation into the $3 / 2$ spin-orbit level would violate the $\Delta J=0, \pm 1$ selection rule for one-photon electronic transitions, so it is not observed.

${ }^{19}$ The relative intensities of the $5 / 2$ level at $564.58 \mathrm{~nm}$ is 210 , while it is 330 for the $7 / 2$ level at $576.52 \mathrm{~nm}$. Avaiable: http://physics.nist.gov/ PhysRefData/ASD/lines_form.html.

${ }^{20}$ X. Y. Cao and M. Dolg, Theor. Chem. Acc. 108(3), 143 (2002).

${ }^{21}$ A. A. Buchachenko, G. Chalasinski, and M. M. Szczesniak, J. Chem. Phys. 131(24), 241102 (2009).

${ }^{22}$ S. L. Laursen and H. E. Cartland, J. Chem. Phys. 95(7), 4751 (1991).

${ }^{23}$ The matrix shift is calculated from the central threefold excitation component of both the thermally stable red and blue sites.

${ }^{24}$ R. Cambi, D. Cappelletti, G. Liuti, and F. Pirani, J. Chem. Phys. 95(3), 1852 (1991).

${ }^{25}$ D. Schwarzhans, and D. Zimmermann, Eur. Phys. J. D 22(2), 193 (2003).

${ }^{26}$ R. Bruhl, J. Kapetanakis, and D. Zimmermann, J. Chem. Phys. 94(9), 5865 (1991).

${ }^{27}$ J. P. Visticot, P. de Pujo, J. M. Mestdagh, A. Lallement, L. Berlande, O. Sublemontier, P. Meynadier, and J. Cuvellier, J. Chem. Phys. 100, 158 (1994).

${ }^{28}$ Molecular dynamics simulations of barium atoms [this work] and more recently sodium atoms [5] isolated in single vacancy sites of $h c p$ or $f c c$ structures of the solid rare gases yielded essentially identical absorption spectra. On this basis, and the fact that a single site is present in Xe, local environments are not considered responsible for the occurrence of pairs of sites for Eu atoms in $\mathrm{Ar}$ and $\mathrm{Kr}$.

${ }^{29}$ B. S. Tsukerblatt, Group Theory in Chemistry and Spectroscopy (Dover, New York, 2006), p. 258.

${ }^{30}$ C. W. Struck and W. H. Fonger, Understanding Luminscence Spectra and Efficiency Using Wp and Related Functions (Springer-Verlag, Berlin, 1991).

${ }^{31}$ I. Bersuker, The Jahn-Teller Effect (Cambridge University Press, Cambridge, 2006)

${ }^{32}$ The intensity data for gas phase $\mathrm{Eu}$ was obtained from NIST at: http://physics.nist.gov/PhysRefData/ASD/lines_form.html.

${ }^{33}$ H. E. Hallam, Vibrational Spectroscopy of Trapped Species (Wiley, New York, 1973). 